\title{
Unerwünschte Wirkungen von Biologika bei Psoriasis
}

\author{
Stephen J. Lockwood ${ }^{\mathrm{a}}$ Lisette M. Prens ${ }^{\mathrm{a}} \quad$ Alexa B. Kimball $^{\mathrm{b}}$ \\ a Department of Dermatology, Clinical Unit for Research Trials in Skin, Massachusetts General Hospital, Harvard Medical School, \\ Boston, MA, USA; \\ ${ }^{b}$ Beth Israel Deaconess Medical Center, Harvard Medical School, Boston, MA, USA
}

\section{Zusammenfassung}

Psoriasis ist eine chronische Autoimmunerkrankung, von der weltweit Millionen Menschen betroffen sind. Die Erkrankung ist nicht nur für sich selbst genommen sehr belastend und kann die Lebensqualität verringern, sondern sie stellt auch einen Risikofaktor für weitere systemische Erkrankungen dar, z.B. metabolisches Syndrom, kardiovaskuläre und maligne Erkrankungen. In der Behandlung der Psoriasis wurden im mittleren bis späten 20. Jahrhundert bahnbrechende Fortschritte erzielt, unter anderem durch die Entwicklung von topischen Kortikosteroiden und Vitamin-D-Analoga sowie Methotrexat, systemischen Retinoiden und Phototherapie. Doch erst 2004, mit dem Aufkommen der Biologika - neuartiger systemischer biologischer Arzneimittel, die sehr spezifisch gegen bestimmte Zielstrukturen des Immunsystems gerichtet sind -, wurde das Ansprechen laut PASI (Psoriasis Area and Severity Index) als Maßstab für das Hauptziel der Behandlung von einer Verbesserung um 50\% (PASI 50) auf PASI 75, PASI 90 und sogar PASI 100, d.h. die vollständige Rückbildung der Hautläsionen, angehoben. Heute er- reichen viele Patienten mit einer Biologika-Therapie routinemäßig eine Verringerung der kutanen Krankheitslast um 75\% oder 90\% und eine signifikante Verbesserung ihrer Lebensqualität insgesamt. Biologika sind im Allgemeinen gut verträglich und sicher, doch wie jedes Arzneimittel sind auch sie mit unerwünschten Wirkungen assoziiert. Einige dieser unerwünschten Wirkungen können anhand von Effekten der Immunmodulation, von Tiermodellen sowie von menschlichen Populationen mit bekannten Zytokindefizienzen vorhergesagt werden. Im nächsten Schritt ist es wichtig, sowohl in klinischen Studien als auch in Registern zur Anwendungsbeobachtung die Sicherheitsprofile dieser Wirkstoffe sorgfältig zu überwachen, um die Langzeitsicherheit zu bestätigen. Erfreulicherweise bescheinigen die großen Sicherheitsregister den neu hinzugekommenen bzw. hinzukommenden Biologika ein verbessertes Sicherheitsprofil.

C 2017 S. Karger GmbH, Freiburg

\section{Einleitung}

Psoriasis ist eine komplexe inflammatorische Autoimmunerkrankung, von der rund 1-3\% der Allgemeinbevölkerung betroffen sind, und die Inzidenz scheint weiter zu steigen [1-4]. Die Krankheit tritt bei Männern und Frauen gleich häufig auf, meist im zweiten oder vierten Lebensjahrzehnt [5]. Man unterscheidet verschiedene Unterformen der Psoriasis, darunter Plaque-Psoriasis, Psoriasis guttata, erythrodermische Psoriasis, Psoriasis pustulosa, Nagelpsoriasis und Psoriasis-Arthritis. Die chronische Psoriasis vom Plaque-Typ ist die häufigste Form der Psoriasis, auf sie entfallen rund 79\% der Erkrankungen im Erwachsenenalter und 74\% der Fälle, die im Kindes- und Jugendalter auftreten [4].
Psoriasis ist jedoch nicht nur eine chronische Erkrankung, sondern auch ein Risikofaktor für weitere systemische Erkrankungen wie Diabetes, Hypertonie, metabolisches Syndrom, chronisch-entzündliche Darmerkrankung (CED), kardiovaskuläre Erkrankungen, maligne Erkrankungen, Infektionen sowie weitere Autoimmunerkrankungen [6-10]. Die Gründe für diese Assoziationen sind noch nicht vollständig geklärt, könnten jedoch zumindest teilweise in den Auswirkungen immunvermittelter chronischer Entzündungen, Begleiterkrankungen, der Lebensführung sowie Nebenwirkungen von systemischen Therapien liegen. Psoriasis bedeutet eine erhebliche Einschränkung der Lebensqualität für die Betroffenen und zugleich eine hohe wirtschaftliche Belastung für das Gesundheitssystem [11, 12].

\section{KARGER}

Fax +497614520714

information@karger.com

www.karger.com

\section{(c) 2017 S. Karger GmbH, Freiburg}

Accessible online at:

www.karger.com/kkd
Alexa B. Kimball, MD, MPH

Beth Israel Deaconess Medical Center

Harvard Medical School

Boston, MA 02215, USA

KimballMDresearch@Gmail.com 


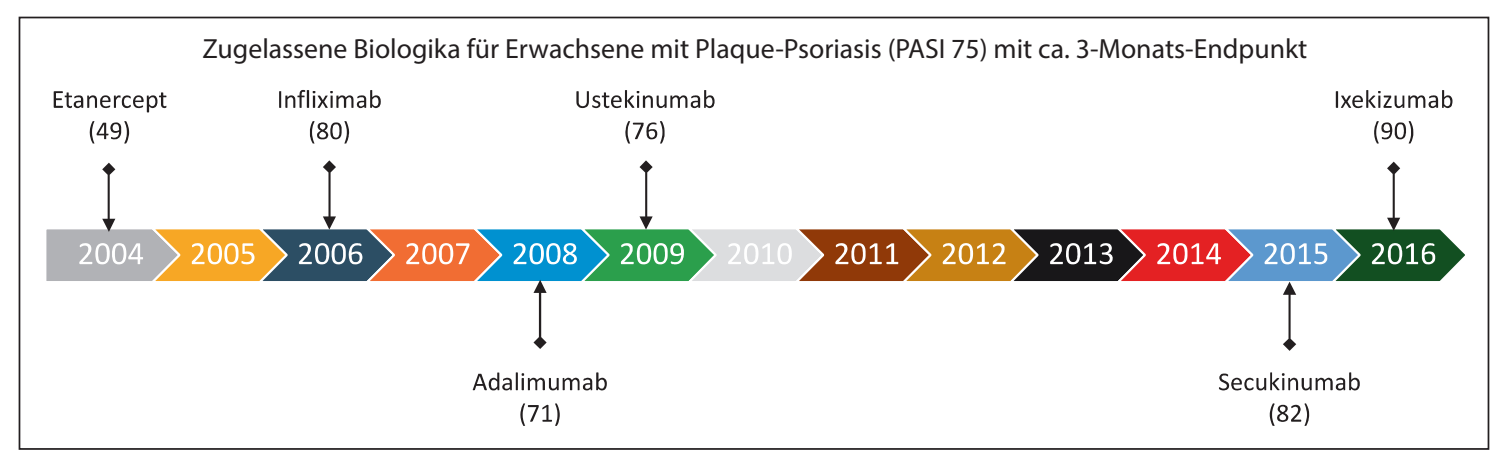

An der Pathophysiologie der Psoriasis sind T-Lymphozyten, dendritische Zellen, Makrophagen, neutrophile Granulozyten sowie Keratinozyten beteiligt. Man nimmt an, dass Antigenreize plasmazytoide dendritische Zellen und andere Immunzellen in der Haut aktivieren [13]. Der Tumornekrosefaktor-alpha (TNF- $\alpha$ ), der von aktivierten dendritischen Zellen und anderen Zellen der angeborenen Immunabwehr produziert wird, regt seinerseits die Aktivierung myeloischer dendritischer Zellen in der Haut an, die Interleukin(IL-)23 und IL-12 produzieren. IL-23 fördert die Entwicklung von Th17-Zellen, die eine zentrale Rolle in der Pathogenese der Psoriasis spielen, indem sie IL-17A und IL-22 ausschütten, was wiederum die Keratinozyten zur Vermehrung anregt. TNF- $\alpha$ fördert ebenfalls die Vermehrung der Keratinozyten und die Adhäsion der Zellen untereinander sowie das Wachstum und die Invasivität dermaler Fibroblasten in Psoriasis-Läsionen [14]. Diese epidermale Hyperproliferation mit anomaler Differenzierung, inflammatorischer dermaler Infiltration und veränderter Gefäßversorgung führt zur kutanen Manifestation der erythematösen Plaques mit silbrigen Schuppen [13]. Wenn eine PsoriasisErkrankung abklingt, sinkt in der betroffenen Haut die Zahl der T-Lymphozyten, der dermalen dendritischen Zellen und der Neutrophile ebenso wie die Konzentration von TNF- $\alpha$, IFN- $\alpha$, IL-12, IL-23 und IL-17 infolge geringerer Expression der entsprechenden Gene [15].

\section{Eine Revolution in der Psoriasis-Therapie}

Bis zum 19. Jahrhundert war Psoriasis eine weitgehend unerforschte Erkrankung, und die Behandlungsmöglichkeiten waren begrenzt. Das 1780 in die Medizin eingeführte Arsen wurde zu einer der ersten systemischen Behandlungen, die bei Psoriasis angewandt wurden [16-18]. Trotz erheblicher Nebenwirkungen von Organpigmentierung bis hin zur Entwicklung maligner Tumoren war Arsen noch bis zur Mitte des 20. Jahrhunderts in der Behandlung der Psoriasis gebräuchlich [19]. 1895 setzte Brault Quecksilber-Injektionen zur Behandlung von Psoriasis-Arthritis ein [20]. Psoriasis wurde weithin als Infektionskrankheit angesehen, und man experimentierte mit antibakteriellen Therapien, die jedoch wenig Nutzen brachten [21].

Zu Hippokrates Zeiten wurden häufig Teerpräparate zur Behandlung verschiedener Hauterkrankungen eingesetzt. Teer wirkt antiproliferativ und antiinflammatorisch, reizt jedoch die Haut und riecht unangenehm. Teer wurde zu Salben, Pasten und Ölen ver- arbeitet, die direkt auf die Haut aufgetragen oder als Badezusatz verwendet wurden [22]. Es ist nachgewiesen, dass Steinkohlenteer die Schwere der Erkrankung um ein gewisses Maß reduziert. In den 1920er Jahren berichtete Alderson von einer Besserung der Psoriasis durch ultraviolette Bestrahlung, und 1925 setzte Goeckerman Teer in Kombination mit ultravioletter Bestrahlung ein [23, 24]. 1973 wiesen Tronnier und Schule [25] eine Besserung der Psoriasis nach topischer Anwendung von Psoralenen und UVA-Strahlung (PUVA) nach.

Mitte des 20. Jahrhunderts wurden Kortikosteroide in die Psoriasis-Therapie eingeführt. Große Fortschritte brachte in den 1950er Jahren das Aufkommen von Methotrexat (MTX), mit nachweislich zufriedenstellendem Ansprechen bei Patienten mit mittel- bis hochgradiger Psoriasis und Psoriasis-Arthritis [26]. Cyclosporin kam als hochwirksame Therapieoption hinzu, doch die Nephrotoxizität als Nebenwirkung beschränkt die langfristige Nutzbarkeit [27]. In den 1970er Jahren wurde das therapeutische Arsenal um systemische Retinoide, Isotretinoin und Acitretin erweitert [28]. Vitamin-D-Analoga kamen hinzu und bewirkten eine Verlangsamung der Epidermopoese und Stimulation der Keratinozytendifferenzierung [29].

Erst das letzte Jahrzehnt jedoch brachte bahnbrechende Fortschritte in der Behandlung der mittel- bis hochgradigen Psoriasis vom Plaque-Typ in Form der neuartigen systemischen biologischen Arzneimittel oder Biologika (Abb. 1).

Im Gegensatz zu den herkömmlichen, unspezifischen Immunsuppressiva richten sich Biologika gezielt gegen die Komponenten des Immunsystems, die an den pathophysiologischen Prozessen der Erkrankung beteiligt sind. Diese neueren Arzneimittel haben das Ansprechen laut PASI (Psoriasis Area and Severity Index) als Maßstab für das Hauptziel der Behandlung von einer Verbesserung um 50\% (PASI 50) auf PASI 75, PASI 90 und sogar PASI 100, d.h. die vollständige Rückbildung von Hautläsionen, erhöht. 2013 wurde bereits fast jeder vierte Patient mit mittel- bis hochgradiger Psoriasis mit Biologika behandelt, in den meisten Fällen Adalimumab oder Etanercept [30].

Etanercept wurde 2004 für die Behandlung der mittel- bis hochgradigen Psoriasis zugelassen; seitdem hat die US-Aufsichtsbehörde FDA noch mehreren weiteren TNF- $\alpha$-Inhibitoren die Zulassung erteilt. 2009 kam Ustekinumab auf den Markt, das am IL-12/23-Signalweg ansetzt [31]. 2015 bzw. 2016 kamen Secukinumab und Ixekizumab hinzu, zwei gegen den IL-17-Signalweg gerichtete Wirkstoffe, die das therapeutische Wirksamkeitspo- 
tenzial weiter erhöhten. Zusätzlich befinden sich weitere Biologika in der Entwicklung, die besonders genau auf ihre Zielmoleküle wie IL-23p19 oder IL-17-Rezeptoren zugeschnitten sind, sowie Biosimilars von TNF- $\alpha$-Inhibitoren. Welcher Wirkstoff letztlich für welchen Patienten am besten geeignet ist, wird durch verschiedene Faktoren mitbestimmt - von den Begleiterkrankungen und dem Psoriasis-Subtyp des Patienten über das Sicherheitsprofil des Arzneimittels bis hin zur Präferenz des Patienten.

\section{Biologika zur Behandlung von Psoriasis: Wirkmechanismen und Wirksamkeit}

Mit dem Aufkommen der Biologika hat sich das therapeutische Management von Patienten mit mittel- bis hochgradiger Psoriasis von Grund auf verändert. Ihre Wirksamkeit im Vergleich zu konventionellen systemischen Therapien ist in klinischen Studien nachgewiesen. Neue Biologika wie Secukinumab und Ixekizumab sind noch spezifischer gegen Entzündungsmediatoren gerichtet, die in nachgelagerten inflammatorischen Prozessen eine Schlüsselrolle für den Fortbestand der Psoriasis-Erkrankung spielen. Damit werden weitere Fortschritte in der Wirksamkeit der Behandlung erzielt. In den weiterführenden Tabellen sind die Wirkmechanismen und Wirksamkeitsdaten aus klinischen Studien für die verschiedenen Biologika-Klassen zusammengefasst: TNF- $\alpha$-Inhibitoren (Tab. 1), IL-12/23-Inhibitoren (Tab. 2) und IL-17-Inhibitoren (Tab. 3; Anm. d. Red.: Die Tabellen 1-3 und die Textverweise zu den Referenzen 32-50 sind im englischen Originalbeitrag unter (www.karger.com/Book/Home/276505) zu finden).

\section{Sicherheit von Biologika}

\section{Überblick}

Die gezielte Neutralisierung bestimmter Zytokine durch Biologika hat die therapeutische Wirksamkeit und die Behandlungsergebnisse bei verschiedenen immunvermittelten Krankheiten, darunter Psoriasis, signifikant verbessert. Diese Biologika sind im Allgemeinen sicher und gut verträglich, doch wie jedes Arzneimittel können auch sie mit unerwünschten Wirkungen einhergehen. Einige dieser Risiken können anhand allgemeiner Erkenntnisse über die Effekte der Immunmodulation, andere wiederum anhand von Tiermodellen sowie von menschlichen Populationen mit bekannten Zytokindefizienzen vorhergesagt werden. So unterliegen Personen mit IL-17-Defizit einem erhöhten Risiko für CandidaInfektionen - ein Problem, das auch bei Psoriasis-Patienten unter Behandlung mit IL-17-Inhibitoren besteht (Abb. 2) (Anm. d. Red.: Abbildung 2 finden Sie im englischen Originalbeitrag unter www.karger.com/Book/Home/276505) [51]. Personen mit IL-23-
Defizit hingegen sind besonders anfällig für Salmonellen, was sich bei Patienten unter IL-23-Inhibitoren bisher nicht als Problem 0manifestiert hat.

Die wachsenden Bestände an Sicherheitsdaten zu allen diesen Wirkstoffen, die in Registern (PSOLAR, BADBIR, PsoBest) gesammelt werden und inzwischen Tausende Patienten über lange Nachbeobachtungzeiträume abdecken, stimmen weitgehend zuversichtlich [52-56]. Zu den Risiken, die grundsätzlich mit Biologika verbunden sind, zählen unerwünschte Arzneimittelwirkungen, erhöhtes Infektionsrisiko, Entwicklung von Neoplasien wie z.B. nicht-melanomatösem Hautkrebs, Verschlechterung zugrunde liegender bzw. Reaktivierung latenter Erkrankungen sowie hämatologische Störungen. Patienten, die solche Arzneimittel erhalten, müssen engmaschig überwacht werden. Bevor eine Behandlung mit Biologika begonnen wird, ist sorgfältig zu prüfen, ob alle Immunisierungen vorliegen, die für den Patienten in seinem Alter gemäß aktueller Leitlinien empfohlen werden, da Lebendimpfstoffe unter Biologika-Therapie zu vermeiden sind. Die Anwendung von Biologika, insbesondere in Kombination mit MTX, kann das Reaktivierungsrisko einer Infektion mit verschiedenen Viren erhöhen, darunter das Varicella-Virus, der Erreger von Herpes zoster [57].

Die Risiken variieren zwar je nach Altersgruppe, doch typischerweise erfolgt bei allen Patienten ein Screening auf Tuberkulose (TB), Hepatitis-B- und -C-Virus sowie gegebenenfalls den HIVStatus, bevor eine Biologika-Therapie begonnen wird [58]. Grundsätzlich sollte bei Patienten mit chronischer Hepatitis-B-Infektion, die keine signifikante Leberschädigung aufweisen, vor einer Biologika-Therapie eine antivirale Therapie erfolgen [59]. Bei Patienten mit akuter Hepatitis-C-Infektion oder signifikanter Leberschädigung sind Biologika zu vermeiden, wenn dies klinisch angemessen ist. Bei Patienten mit aktiver oder latenter TBInfektion ist eine angemessene TB-Therapie durchzuführen, bevor oder während die Biologika-Therapie eingeleitet wird (je nach Wirkstoff). Im Vergleich zur Psoriasis-Therapie mit nicht-biologischen systemischen Wirkstoffen wurde bisher keine signifikante Assoziation zwischen Biologika und schwerwiegenden kardiovaskulären unerwünschten Ereignissen (MACE), malignen Erkrankungen oder Todesfällen über das für die Psoriasispopulation insgesamt erwartbare Maß hinaus festgestellt [60].

Den vollständigen englischsprachigen Beitrag «Adverse Reactions to Biologics in Psoriasis» von Lockwood SJ, Prens LM, Kimball AB finden Sie ab Ende Oktober 2017 unter www.karger.com/Book/Home/276505. 
1 Ludwig RJ, Herzog C, Rostock A, et al: Psoriasis: a possible risk factor for development of coronary artery calcification. Br J Dermatol 2007;156:271-276.

2 Gelfand JM, Weinstein R, Porter SB, Neimann AL, Berlin JA, Margolis DJ: Prevalence and treatment of psoriasis in the United Kingdom: a populationbased study. Arch Dermatol 2005; 141:1537-1541.

3 Icen M, Crowson CS, McEvoy MT, Dann FJ, Gabriel SE, Maradit Kremers H: Trends in incidence of adult-onset psoriasis over three decades: a populationbased study. J Am Acad Dermatol 2009;60:394-401.

4 Tollefson MM, Crowson CS, McEvoy MT, Maradit Kremers H: Incidence of psoriasis in children: a population-based study. J Am Acad Dermatol 2010;62:979-987.

5 Mervic L: Management of moderate to severe plaque psoriasis in pregnancy and lactation in the era of biologics. Acta Dermatovenerol Alp Pannonica Adriat 2014;23:27-31.

6 Gelfand JM, Neimann AL, Shin DB, Wang X, Margolis DJ, Troxel AB: Risk of myocardial infarction in patients with psoriasis. JAMA 2006; 296:1735-1741.

7 Gisondi P, Tessari G, Conti A, et al: Prevalence of metabolic syndrome in patients with psoriasis: a hospital-based case-control study. $\mathrm{Br} \mathrm{J}$ Dermatol 2007; 157:68-73.

8 Neimann AL, Shin DB, Wang X, Margolis DJ, Troxel AB, Gelfand JM: Prevalence of cardiovascular risk factors in patients with psoriasis. J Am Acad Dermatol 2006;55:829-835.

9 Wu JJ, Nguyen TU, Poon KY, Herrinton LJ: The association of psoriasis with autoimmune diseases. J Am Acad Dermatol 2012;67:924-930.

10 Wakkee M, de Vries E, van den Haak P, Nijsten $\mathrm{T}$ : Increased risk of infectious disease requiring hospitalization among patients with psoriasis: a populationbased cohort. J Am Acad Dermatol 2011;65:1135-1144.

11 Rapp SR, Feldman SR, Exum ML, Fleischer AB Jr, Reboussin DM: Psoriasis causes as much disability as other major medical diseases. J Am Acad Dermatol 1999;41:401-407.

12 Vanderpuye-Orgle J, Zhao Y, Lu J, et al: Evaluating the economic burden of psoriasis in the United States. J Am Acad Dermatol 2015;72: 961-967.e5.

13 Nestle FO, Kaplan DH, Barker J: Psoriasis. N Engl J Med 2009;361:496-509.

14 Menter A, Feldman SR, Weinstein GD, et al: A randomized comparison of continuous vs. intermittent infliximab maintenance regimens over 1 year in the treatment of moderate-tosevere plaque psoriasis. J Am Acad Dermatol 2007;56:31.e1-e15.

15 Menter A, Tyring SK, Gordon K, et al: Adalimumab therapy for moderate to severe psoriasis: a randomized, controlled phase III trial. J Am Acad Dermatol 2008;58:106-115.

16 Fowler T: Medical Reports on the Effects of Arsenic in. London Med J 1786, pp 192-205.

17 PE B: Psoriasis. A brief historical review. Arch Derm Syph 1936;33:327-334.

18 Bechet P: History of the use of arsenic in dermatology. Arch Dermatol 1931;23:110-117.
19 Gruber F, Kastelan M, Brajac I: Psoriasis treatment-yesterday, today, and tomorrow. Acta Dermatovenerol Croat 2004;12:30-34.

20 Brault J: Deux cas de psoriasis traite par les injections mercurielles. Bull Soc Franc Dermatol 1895:332.

21 Wolf R, Feuerman FJ: Failure of osmotic diuresis as therapy for psoriasis. Arch Dermatol 1983;119:95.

22 Menter A, Stoff B: Psoriasis. London, Manson Publishing Ltd., 2010.

23 Alderson HE: Heliotherapy for psoriasis. Arch Dermatol 1923;8:79-80.

24 Moscaliuc ML, Heller MM, Lee ES, Koo J: Goeckerman therapy: a very effective, yet often forgotten treatment for severe generalized psoriasis. J Dermatolog Treat 2013;24:34-37.

25 Tronnier H, Schule D: In Book of Abstracts, Symposia and Contributed Papers, 6th International Congress of Photobiology. Bochum, August 21-25, 1972.

26 Edmundson WF, Guy WB: Treatment of psoriasis with folic acid antagonists. AMA Arch Derm 1958;78:200-203.

27 Mueller W, Herrmann H: Ciclosporin A for psoriasis. N Engl J Med 1979;301:555.

28 Orfanos CE, Runne U: Systemic use of a new retinoid with and without local dithranol treatment in generalized psoriasis. Br J Dermatol 1976;95:101-103

29 Smith EL, Walworth NC, Holick MF: Effect of 1 alpha,25-dihydroxyvitamin D3 on the morphologic and biochemical differentiation of cultured human epidermal keratinocytes grown in serumfree conditions. J Invest Dermatol 1986;86:709-714.

30 Armstrong AW, Robertson AD, Wu J, Schupp C, Lebwohl MG: Undertreatment, treatment trends, and treatment dissatisfaction among patients with psoriasis and psoriatic arthritis in the United States: findings from the national psoriasis foundation surveys, 2003-2011. JAMA Dermatol 2013;149:1180-1185.

31 Manalo IF, Gilbert KE, Wu JJ: Time to raise the bar to psoriasis area severity index 90 and 100 . J Drugs Dermatol 2015;14:1086-1088.

32 Gall JS, Kalb RE: Infliximab for the treatment of plaque psoriasis. Biologics 2008;2:115-124.

33 Nast A, Sporbeck B, Rosumeck S, et al: Which antipsoriatic drug has the fastest onset of action? Systematic review on the rapidity of the onset of action. J Invest Dermatol 2013;133: 1963-1970.

34 Reich K, Nestle FO, Papp K, et al: Infliximab induction and maintenance therapy for moderate-to-severe psoriasis: a phase III, multicentre, double-blind trial. Lancet 2005;366: 1367-1374.

35 Barker J, Hoffmann M, Wozel G, et al: Efficacy and safety of infliximab vs. methotrexate in patients with moderateto-severe plaque psoriasis: results of an open-label, active-controlled, randomized trial (RESTORE1). Br J Dermatol 2011;165:1109-1117.

36 Saurat JH, Stingl G, Dubertret L, et al: Efficacy and safety results from the randomized controlled comparative study of adalimumab vs. methotrexate vs. placebo in patients with psoriasis (CHAMPION). Br J Dermatol 2008;158: $558-566$
37 Leonardi C, Langley RG, Papp K, et al: Adalimumab for treatment of moderate to severe chronic plaque psoriasis of the hands and feet: efficacy and safety results from REACH, a randomized, placebo-controlled, double-blind trial. Arch Dermatol 2011;147:429-436.

38 Bissonnette R, Bolduc C, Poulin Y, Guenther L, Lynde CW, Maari C: Efficacy and safety of adalimumab in patients with plaque psoriasis who have shown an unsatisfactory response to etanercept. J Am Acad Dermatol 2010;63:228-234.

39 Nguyen TU, Koo J: Etanercept in the treatment of plaque psoriasis. Clin Cosmet Investig Dermatol 2009;2:77-84.

40 Leonardi CL, Powers JL, Matheson RT, et al: Etanercept as monotherapy in patients with psoriasis. N Engl J Med 2003;349:2014-2022.

41 Tyring S, Gottlieb A, Papp K, et al: Etanercept and clinical outcomes, fatigue, and depression in psoriasis: doubleblind placebo-controlled randomized phase III trial. Lancet 2006;367:29-35.

42 Benson JM, Peritt D, Scallon BJ, et al: Discovery and mechanism of ustekinumab: a human monoclonal antibody targeting interleukin-12 and interleukin-23 for treatment of immunemediated disorders. MAbs 2011;3:535-545.

43 Papp KA, Langley RG, Lebwohl M, et al: Efficacy and safety of ustekinumab, a human interleukin-12/23 monoclonal antibody, in patients with psoriasis: 52-week results from a randomised, double-blind, placebo-controlled trial (PHOENIX 2). Lancet 2008;371:1675-1684.

44 Leonardi CL, Kimball AB, Papp KA, et al: Efficacy and safety of ustekinumab, a human interleukin-12/23 monoclonal antibody, in patients with psoriasis: 76-week results from a randomised, double-blind, placebo-controlled trial (PHOENIX 1). Lancet 2008;371:1665-1674.

45 Kimball AB, Gordon KB, Fakharzadeh S, et al: Long-term efficacy of ustekinumab in patients with moderate-to-severe psoriasis: results from the PHOENIX 1 trial through up to 3 years. Br J Dermatol 2012;166:861-872.

46 Griffiths CE, Strober BE, van de Kerkhof P, et al: Comparison of ustekinumab and etanercept for moderate-to-severe psoriasis. N Engl J Med 2010;362:118-128.

47 Langley RG, Elewski BE, Lebwohl M, et al: Secukinumab in plaque psoriasis-results of two phase 3 trials. N Engl J Med 2014;371: 326-338.

48 Blauvelt A, Reich K, Tsai TF, et al: Secukinum$\mathrm{ab}$ is superior to ustekinumab in clearing skin of subjects with moderate-to-severe plaque psoriasis up to 1 year: Results from the CLEAR study. J Am Acad Dermatol 2017;76:60-69.e9.

49 Griffiths CE, Reich K, Lebwohl M, et al: Comparison of ixekizumab with etanercept or placebo in moderate-to-severe psoriasis (UNCOVER-2 and UNCOVER-3): results from two phase 3 randomised trials. Lancet 2015; 386:541-551

50 Gordon KB, Blauvelt A, Papp KA, et al: Phase 3 trials of ixekizumab in moderate-to-severe plaque psoriasis. N Engl J Med 2016;375: 345-356.

51 Blauvelt A, Lebwohl MG, Bissonnette R: IL-23/ IL-17A dysfunction phenotypes inform possible clinical effects from anti-IL-17A therapies. J Invest Dermatol 2015;135:1946-1953. 
52 Augustin M, Spehr C, Radtke MA, et al: German psoriasis registry PsoBest: objectives, methodology and baseline data.J Dtsch Dermatol Ges 2014;12:48-57.

53 Papp KA, Strober B, Augustin M, et al: PSOLAR: design, utility, and preliminary results of a prospective, international, disease-based registry of patients with psoriasis who are receiving, or are candidates for, conventional systemic treatments or biologic agents. J Drugs Dermatol 2012;11:1210-1217.

54 US National Institute of Health: A Multicenter Open Registry of Patients with Plaque Psoriasis Who Are Candidates for Systemic Therapy Including Biologics.Bethesda, National Library of Medicine (US), 2007 (cited January 23, 2017).
55 Iskandar IY, Ashcroft DM, Warren RB, et al: Patterns of biologic therapy use in the management of psoriasis: cohort study from the British Association of Dermatologists Biologic Interventions Register (BADBIR). Br J Dermatol 2017;176:1297-1307.

56 Reich K, Mrowietz U, Radtke MA, et al: Drug safety of systemic treatments for psoriasis: results from the German Psoriasis Registry PsoBest. Arch Dermatol Res 2015;307:875-883.

57 Shalom G, Zisman D, Bitterman H, et al: Systemic therapy for psoriasis and the risk of Herpes Zoster: a 500,000 personyear study. JAMA Dermatol 2015;151:533-538.
58 Wendling D, Auge B, Bettinger D, et al: Reactivation of a latent precore mutant hepatitis $B$ virus related chronic hepatitis during infliximab treatment for severe spondyloarthropathy. Ann Rheum Dis 2005;64:788-789.

59 Singh JA, Saag KG, Furst D: Reply: to PMID 22473917. Arthritis Care Res (Hoboken) 2013; 65:832-833.

60 Gottlieb AB, Kalb RE, Langley RG, et al: Safety observations in 12095 patients with psoriasis enrolled in an international registry (PSOLAR): experience with infliximab and other systemic and biologic therapies. J Drugs Dermatol 2014;13:1441-1448. 